\title{
Effect of a Nutritionally Balanced Diet Comprising Whole Grains and Vegetables Alone or in Combination with Probiotic Supplementation on the Gut Microbiota
}

\author{
Young Joo $\mathrm{Oh}^{1,2}$, Kisun Nam ${ }^{1}$, Yanghee Kim¹, Seo Yeon Lee ${ }^{1}$, Hyung Sook Kim ${ }^{3}$, Jung II Kang ${ }^{1}$, \\ Sang Yun Lee', and Keum Taek Hwang ${ }^{2}$ \\ ${ }^{1}$ Pulmuone Co., Ltd., Seoul 06367, Korea \\ ${ }^{2}$ Department of Food and Nutrition, and Research Institute of Human Ecology, Seoul National University, Seoul 08826, Korea \\ ${ }^{3}$ Department of Food and Nutrition, The University of Suwon, Gyeonggi 18323, Korea
}

\begin{abstract}
Dysbiosis is a microbial imbalance, which often causes diseases and can be triggered by diet. Here, we determined the effect of a nutritionally balanced diet rich in vegetables and whole grains alone and/or in combination with probiotics on the gut microbiota of healthy adults. We conducted a parallel-group randomized trial enrolling 63 healthy participants who were administered either a balanced diet (B-diet group), a probiotic capsule containing Lactobacillus plantarum PMO 08 (probiotics group), or a balanced diet plus probiotic capsule (synbiotics group) once daily for 2 weeks. The gut microbiota of each participant was analyzed via 16S ribosomal RNA MiSeq-based sequencing. Gastrointestinal symptoms and defecation habits were evaluated using questionnaires. The B-diet group showed significantly reduced Firmicutes-toBacteroidetes ratio $(P<0.05)$ and abundances of the genera Blautia $(P<0.01)$, Dorea $(P<0.05)$, and Lachnoclostridium $(P<$ 0.05). Furthermore, the abundance of Bacteroides increased $(P<0.05)$ compared to baseline levels. In the synbiotics group, Lactobacillus abundance increased significantly $(P<0.05)$ and defecation difficulty decreased $(P<0.05)$, confirming a synergistic effect of combined intake. All groups showed a significant reduction in the abundance of Clostridiaceae $(P<0.001)$ and alleviation of bloating symptoms $(P<0.05)$. Moreover, the relative abundance of Faecalibacterium significantly increased in the probiotics group $(P<0.05)$. Therefore, the individual or combined intake of a nutritionally balanced diet and $L$. plantarum PMO 08 beneficially modifies the gut microbiota with the potential to alleviate gastrointestinal symptoms and improve defecation habits.
\end{abstract}

Keywords: Firmicutes-to-Bacteroidetes ratio, gut health, gut microbiota, Lactobacillus plantarum PMO 08, nutritionally balanced diet

\section{INTRODUCTION}

The intestinal ecosystem is closely related to human health. Intestinal dysbiosis is associated with various diseases, such as inflammatory bowel disease (IBD), obesity, non-alcoholic liver disease, and high blood pressure (Singh et al., 2017). The composition of the gut microbiota is dependent on internal factors, such as sex, race, and age, and is affected by external environmental factors, such as antibiotics, drugs, stress, and diet. Diet is a source of nutrients for microorganisms and is considered the most important factor influencing the gut microbiota (Shin et al., 2019). Arumugam et al. (2011) introduced the following three enterotypes of the human gut micro- biome according to dietary habits: the Bacteroides enterotype in individuals with a high intake of protein and animal fats; the Prevotella enterotype in individuals with a high intake of fiber; and the Ruminococcus enterotype, which is similar to the Bacteroides enterotype but characterized by fast sugar absorption and a greater likelihood of developing obesity. According to a previous study that analyzed the gut microbiota of immigrants from Thailand to the USA, changes in diet alter the ecological environment of the intestines. In particular, Prevotella may replace Bacteroides, which gradually decreases the ability to digest dietary fiber (Vangay et al., 2018). Since such an intestinal ecosystem can have a strong influence on human physiology by interacting with metabolites or symbi- 
otic microorganisms produced by other microorganisms, the abundance of different species in the gut microbiota can be used to predict disease and as an indicator for improvement (Hills et al., 2019). Approximately 90\% of Gram-negative Bacteroidetes and Gram-positive Firmicutes reside in the intestines, with a higher proportion of Firmicutes species associated with various diseases, such as obesity, type 2 diabetes, and IBD (Magne et al., 2020). As the human gut microbiome can change with dietary habits, recent research has focused on investigating the relationship between dietary ingredients and functional foods and the gut microbiota (De Angelis et al., 2019).

Modern society is exposed to the "Western-style diet", which is characterized by excessive intake of energy-rich foods, such as fat, cholesterol, and animal protein. This type of diet causes inflammatory conditions, and sustained inflammatory reactions can lead to various diseases, such as obesity, metabolic syndrome, and colon cancer (Cândido et al., 2018). Specifically, chronic inflammation is caused by lipopolysaccharides, a component of the cell wall of Gram-negative bacteria that is closely related to dietary factors, such as animal fat intake (Schoeler and Caesar, 2019). Therefore, the composition of the gut microbiota has been well studied based on investigations of dietary control for preventing chronic diseases (Chae, 2016; Barrett et al., 2018; Garcia-Mantrana et al., 2018; Losasso et al., 2018). Compared to the regular diet, the Mediterranean diet, which comprises olive oil, whole grains, nuts, vegetables, and fruits, among other foods, increases the levels of Bacteroides, Prevotella, Bifidobacterium, and Lactobacillus and decreases the level of Clostridium. Intake of polyphenols, dietary fiber, plant proteins, and polysaccharides from plant foods induces changes in the gut microbiota (Garcia-Mantrana et al., 2018). An analysis of the gut microbiota based on the dietary patterns of 101 adults in Italy revealed that vegans and vegetarians have higher counts of Bacteroidetes-related operational taxonomic units than omnivores (Losasso et al., 2018). Furthermore, an association analysis of relative bacterial abundance with very low-density lipoprotein (VLDL) in pregnant women revealed that Lachnospiraceae is significantly higher in those consuming a vegetarian diet, and showed a negative correlation between Lachnospira and both VLDL cholesterol and triglyceride levels (Barrett et al., 2018). In addition, a previous study evaluated the effect of a Korean Dietary Approaches to Stop Hypertension (DASH) diet on metabolic syndrome (low-salt diet consisting of grains, vegetables, and fruits) for 10 days. The Korean DASH diet was positively correlated with a reduction in the Firmicutes-to-Bacteroidetes ratio, insulin, and homeostatic model assessment of insulin resistance (Chae, 2016).

Based on recent findings of probiotic function, studies are being conducted on the potential of probiotics for the treatment and prevention of chronic diseases (Shokryazdan et al., 2017). Probiotics are defined as living microorganisms that have a beneficial effect on the host when consumed in adequate amounts. Many of their beneficial effects, such as normalization of intestinal microbiota, competitive exclusion of pathogens, regulation of the intestinal transit, and modulation of the immune response, have been reported (FAO/WHO, 2001; Lee et al., 2018). This study included Lactobacillus plantarum PMO 08, which is isolated from kimchi and has an excellent bile salt hydrolase activity (Yeo et al., 2000). In vitro studies have confirmed that $L$. plantarum PMO 08 has a high acid, salt, and bile resistance; meets the requirements of a probiotic; has a high intestinal viability; and is very adhesive (Jang et al., 2011a; Oh et al., 2020). Furthermore, L. plantarum PMO 08 demonstrates anti-cholesterol properties by regulating the expression of SREBP-2 mRNA, which is involved in cholesterol metabolism, in a high-fat-induced obese mouse model (Oh et al., 2019). In addition, L. plantarum PMO 08 suppresses pruritus in a concentration-dependent manner in a mouse model of histamineor compound 48/80-induced hypersensitivity (Jang et al., 2011b). Although the biomodulatory effect of $L$. plantarum PMO 08 intake has been confirmed in preclinical studies, its effect on the composition of the gut microbiota has not yet been determined.

This study aimed to evaluate the effect of a nutritionally balanced diet consisting of whole grains and vegetables alone or in combination with probiotics on the composition of gut microbiota in healthy adults. Participants were provided with one nutritionally balanced meal per day in the form of a lunch meal box for 2 weeks, and the abundance of major intestinal microorganisms was compared before and after the intervention. Furthermore, $L$. plantarum PMO 08 was provided in the form of a capsule as a probiotics. In addition, the synergistic effects of a synbiotic diet consisting of a nutritionally balanced meal and a probiotic supplement were observed.

\section{MATERIALS AND METHODS}

\section{Subjects and experiment design}

This study enrolled healthy adult volunteers aged 19 65 years from October 8, 2019 to October 22, 2019. All participants provided written informed consent. Participants were randomly assigned to one of three groups and administered either a nutritionally balanced meal (B-diet group), a probiotic capsule (probiotics group), or a nutritionally balanced meal with a probiotic capsule (symbiotic group) once per day for 2 weeks following a 2-week washout period. Feces were collected before and after intake of the experimental diet, and all participants completed questionnaires related to defecation and dietary 


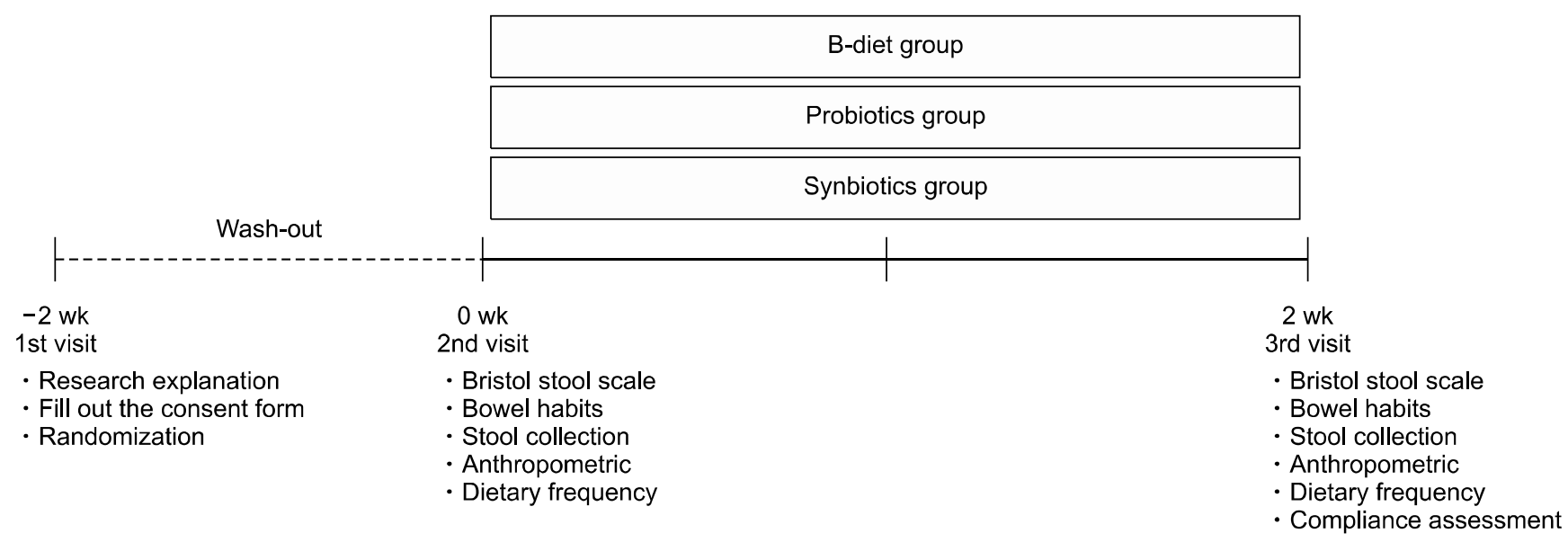

Fig. 1. Schematic outline of the study protocol for the B-diet, probiotics, and synbiotics groups.

habits. Anthropometric biomarkers such as the body weight $(\mathrm{kg})$, body fat percentage (\%), and body mass in$\operatorname{dex}\left(\mathrm{BMI} ; \mathrm{m} / \mathrm{kg}^{2}\right.$ ) were measured at the beginning and end of the intervention periods using a Body Composition Analyzer (InBody 230; InBody Co., Ltd., Seoul, Korea). The overall clinical study design is shown in Fig. 1. This study was conducted with approval of the Institutional Review Board of Suwon University (approval number: 1910-045-01).

\section{Experimental diet}

The nutritionally balanced diet used in the study was a modified version of the 211 diet, a balanced meal plan developed by Pulmuone (Seoul, Korea). The 211 diet emphasizes selection of protein foods with low saturated fats, vegetables, and whole grains to prevent excessive carbohydrate consumption (Ha et al., 2020). Nutritionally balanced meals are rich in whole grains (e.g., oats, barley, quinoa, lentil, and chickpeas), vegetables (e.g., broccoli, carrot, cabbage, tomato, and mushroom), nuts and beans, and have a low content of red meat. The nutritionally balanced diet was high in fiber and low in saturated fats, and was provided in commercialized lunch boxes produced by the Pulmuone Green Juice Co., Ltd. (Chungbuk, Korea). On weekdays, a refrigerated lunch box containing a nutritionally balanced diet was delivered each day, and two frozen lunch boxes were provided on Friday for the weekend. Table 1 shows the nutritional content of each product and a list of the food ingredients. The probiotic capsule $(400 \mathrm{mg})$ containing 10 billion colony forming unit (CFU) of $L$. plantarum PMO 08 was provided by Pulmuone. Participants in the probiotics and synbiotics groups were given the same probiotic capsule, whereas those in the B-diet group were provided with hard capsules of the same formulation as a probiotic supplement filled with maltodextrin instead of probiotics. The capsules were bottled and packaged in sets of 14. Participants were instructed to consume one capsule with wa-
Table 1. Nutrient content of the balanced diet ${ }^{1)}$

\begin{tabular}{lcc}
\hline & $\begin{array}{c}\text { Weekday } \\
\text { (Mon Fri) }\end{array}$ & $\begin{array}{c}\text { Weekend } \\
\text { (Sat } \sim \text { Sun) }\end{array}$ \\
\hline Energy (kcal) & 561 & 592 \\
Carbohydrate (g) & $88(63 \%)^{2)}$ & $84(57 \%)$ \\
Protein (g) & $23(16 \%)$ & $28(19 \%)$ \\
Fat (g) & $13(21 \%)$ & $16(24 \%)$ \\
Dietary fiber (g) & 14 & 4 \\
\hline
\end{tabular}

${ }^{1)}$ Consists of the following: whole grains (oats, sorghum, adlay, quinoa, and brown rice), beans (kidney beans, black beans, lentils, lima beans, chickpeas, pea, and corn), vegetables (eggplant, potato, sweet potato, red pepper, sweet pumpkin, carrot, wild parsley, romaine lettuce, garlic, Japanese apricot, white radish, broccoli, black olive, king oyster mushroom, celery, asparagus, cabbage, lettuce, button mushroom, onion, lotus root, cucumber, red onion, red cabbage, cheongyang chili, red pepper, green pepper, cauliflower, tomato, paprika, zucchini, and fragrant mushroom).

${ }^{2)}$ Percent of calories from each nutrient.

ter once per day. Compliance with intake of a healthy balanced diet with probiotic supplement was investigated using a questionnaire, and participants were instructed to maintain their usual dietary habits until completion of the study.

\section{Assessment of defecation habits}

Defecation habits were evaluated using the Bristol Stool Scale (Lewis and Heaton, 1997) and constipation diagnosis questionnaires (Longstreth et al., 2006), which include stool frequency and regularity, gut transit time, feeling of incomplete evacuation, difficulty in defecation (the act of pushing and squeezing), and bloating, assessed on a four-point scale. Average scores before and after the intervention period were compared.

\section{Analysis of gut microbiota composition}

Fecal samples before and after the intervention period were collected using a stool collection kit (Noble Biosciences, Inc., Gyeonggi, Korea) provided by Green Cross 
Genome Corporation (Gyeonggi, Korea). Collection kits containing fecal samples collected by the subjects were collected and sent to Green Cross Genome Corporation for analysis.

\section{DNA extraction, amplification, and sequencing}

DNA extraction from feces was performed using the Chemagic DNA Stool Kit (PerkinElmer, Inc., Waltham, MA, USA). A NEXTFLEX 16S V4 Amplicon-Seq kit (Bioo Scientific Corp., Austin, TX, USA) was used to create a library of the extracted DNA, which was evaluated for size and quality using the 2200 TapeStation (Agilent Technologies, Inc., Santa Clara, CA, USA). Sequencing was performed using a MiSeq Reagent Kit v2 $(2 \times 150$ bp) and the MiSeq Sequencing System (Illumina, Inc., San Diego, CA, USA).

\section{Bioinformatics analysis}

Pre-processing and amplicon sequence variant clustering of the results file from the Illumina MiSeq Platform were performed using QIIME 2 (https://dev.qiime2.org). For microbial identification, BLCA software (https://github. om/qunfengdong/BLCA) and the NCBI RefSeq database (http://www.ncbi.nlm.nih.gov/refseq) were used. The final processed data were calculated as the number of reads per layer, and each calculated number of reads was converted into relative abundance for analysis.

\section{Statistical analysis}

All results were analyzed using SPSS, version 21.0 software (SPSS Inc., Chicago, IL, USA). Results were expressed as the mean \pm standard deviation (SD) or standard error of mean (SEM). Defecation habits and the relative abundances of gut microbiota before and after the intervention period were verified by paired Student's $t$-tests at $P<0.05, P<0.01$, and $P<0.001$. Statistical significance between experimental groups was analyzed using ANOVA $(P<0.05)$.

\section{RESULTS}

\section{General characteristics of participants}

Table 2 provides information regarding the study participants. A total of 65 individuals were recruited, of which 63 were included in the analysis. The participants had an average age of $36.8 \pm 8.0$ years, average weight of $65.0 \pm$ $2.6 \mathrm{~kg}$, average BMI of $23.7 \pm 0.8 \mathrm{mg} / \mathrm{kg}^{2}$, and average body fat percentage of $30.2 \pm 1.4 \%$. There were no significant differences between groups.

Table 2. General characteristics of study participants

\begin{tabular}{lcccc}
\hline & B-diet group & Probiotics group & Synbiotics group & $P$-value \\
\hline Sex [n (\%)] & & & & $7(33.3)$ \\
Male & $8(38.1)$ & $7(33.3)$ & $14(33.3)$ & 0.995 \\
Female & $13(61.9)$ & $14(66.7)$ & $36.7 \pm 8.4$ & 0.992 \\
Age (yrs) & $36.9 \pm 7.9$ & $65.7 \pm 7.60$ & $65.3 \pm 2.78$ & 0.898 \\
Body weight (kg) & $64.8 \pm 2.60$ & $23.4 \pm 0.64$ & $23.6 \pm 0.78$ & 0.881 \\
BMI (kg/m $\left.{ }^{2}\right)$ & $23.9 \pm 0.84$ & $30.5 \pm 1.41$ & $29.7 \pm 1.56$ & \\
Body fat (\%) & $30.6 \pm 1.22$ & & & \\
\hline
\end{tabular}

Values are expressed as the mean $\pm \mathrm{SD}(\mathrm{n}=21)$.

B-diet group, whole grains and vegetable-enriched diet; probiotics group, probiotic capsule containing Lactobacillus plantarum PMO 08; synbiotics group, whole grains and vegetable-enriched diet combined with L. plantarum PMO 08.

BMI, body mass index.

Table 3. Relative abundances of baseline gut microbiota communities at the phylum level in all participants

\begin{tabular}{lcrrr}
\hline & All subjects $(\mathrm{n}=63)$ & \multicolumn{3}{c}{ Test group ${ }^{\text {NS }}$} \\
\cline { 3 - 5 } & & B-diet group & Probiotics group & Synbiotics group \\
\hline Bacteroidetes & $49.53 \pm 3.84$ & $44.42 \pm 4.23$ & $55.33 \pm 3.25$ & $48.84 \pm 4.05$ \\
Firmicutes & $41.87 \pm 3.37$ & $45.17 \pm 3.51$ & $37.45 \pm 2.85$ & $42.98 \pm 3.75$ \\
Proteobacteria & $3.58 \pm 0.65$ & $4.01 \pm 0.68$ & $4.14 \pm 0.97$ & $2.60 \pm 0.32$ \\
Actinobacteria & $1.03 \pm 0.38$ & $0.87 \pm 0.20$ & $0.89 \pm 0.28$ & $1.32 \pm 0.65$ \\
Fusobacteria & $1.21 \pm 0.97$ & $2.67 \pm 2.30$ & $0.32 \pm 0.25$ & $0.63 \pm 0.35$ \\
Verrucomicrobia & $1.35 \pm 0.98$ & $1.47 \pm 0.98$ & $0.44 \pm 0.32$ & $2.13 \pm 1.64$ \\
Others & $1.36 \pm 0.50$ & $0.99 \pm 0.44$ & $1.43 \pm 0.49$ & $1.66 \pm 0.55$ \\
F/B ratio & $1.15 \pm 0.23$ & $1.34 \pm 0.23$ & $0.80 \pm 0.12$ & $1.31 \pm 0.29$ \\
\hline
\end{tabular}

Values are expressed as the mean \pm SEM $(n=21)$.

F/B ratio, Firmicutes-to-Bacteroidetes ratio. B-diet group, whole grains and vegetable-enriched diet; probiotics group, probiotic capsule containing Lactobacillus plantarum PMO 08; synbiotics group, whole grains and vegetable-enriched diet combined with L. plantarum PMO 08. NS, no significant difference between groups. 


\section{Microbial composition at baseline}

The main phyla analyzed in the feces of all subjects participating in the experiment are shown in Table 3. The gut microbiome of the participants was composed of the

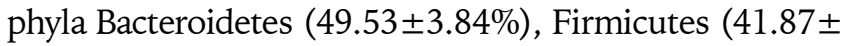
$0.37 \%)$, Proteobacteria (3.58 $\pm 0.65 \%)$, Actinobacteria $(1.03 \pm 0.38 \%)$, Verrucomicrobia $(1.35 \pm 0.98 \%)$, and $\mathrm{Fu}-$ sobacteria $(1.21 \pm 0.97 \%)$, with the top six most abundant phyla accounting for $98.5 \%$ of the microbiota. The ratio of Firmicutes-to-Bacteroidetes (F/B) ratio was $1.15 \pm 0.23$, and there were no significant differences between groups.

\section{Changes in gut microbiota induced by dietary intake}

Changes in the main phyla according to dietary intake are shown in Fig. 2. The B-diet group increased the abundance of Bacteroidetes by $113 \%(P=0.063)$, whereas the abundance of Firmicutes decreased to $84 \%(P<0.01)$. No significant changes were observed in the probiotics and synbiotics groups. The F/B ratio was reduced in the B-diet, probiotics, and synbiotics groups to $71.3 \%, 95.3 \%$, and $88.6 \%$, respectively. In particular, a significant decrease in the $\mathrm{F} / \mathrm{B}$ ratio was observed in the B-diet group compared with baseline ( $P<0.05$; Fig. 3$)$.

Changes in the gut microbiota according to dietary intake at the family level are shown in Fig. 4. The B-diet group showed a significant increase in the abundance of Bacteroidaceae $(P<0.05)$ and a significant decrease in the abundance of Clostridiaceae $(P<0.001)$ and Lachnospiraceae $(P<0.01)$. The probiotics group showed a significant decrease in the relative abundance of Clostridiaceae $(P<$ 0.001 ), and the synbiotics group showed a significant in-

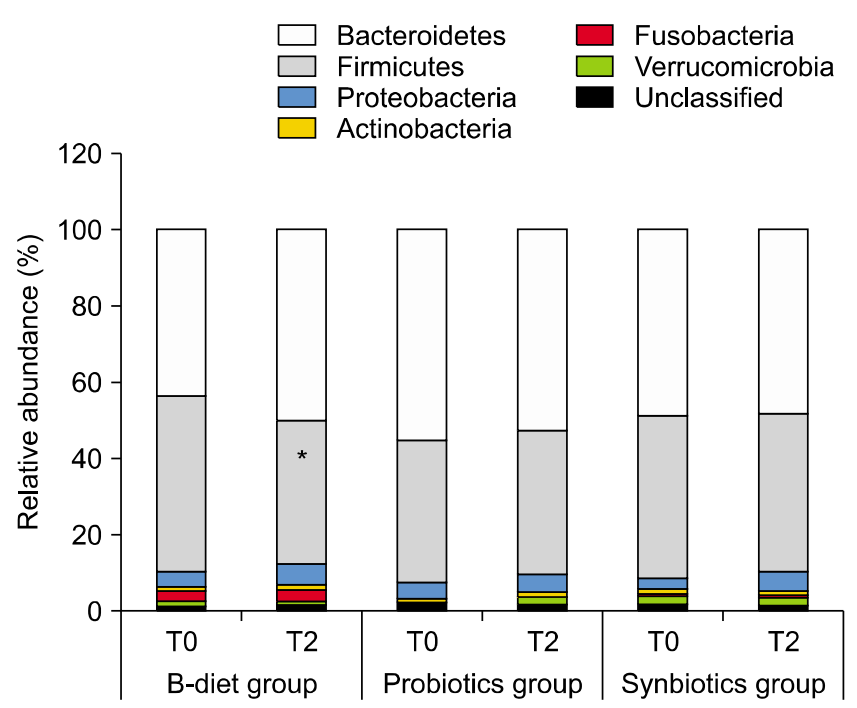

Fig. 2. Relative abundance of bacteria constituting the gut microbiome at the phylum level. T0, baseline value; T2, after 2 weeks of intervention. B-diet group, whole grains and vegetable-enriched diet; probiotics group, probiotics capsule containing Lactobacillus plantarum PMO 08; synbiotics group, whole grains and vegetable-enriched diet combined with L. plantarum PMO 08. * $P<0.05$ compared with baseline (TO), calculated using paired $t$-tests. crease in the relative abundance of Lactobacillaceae $(P<$ $0.05)$ and a significant decrease in the abundance of Clostridiaceae $(P<0.001)$.

Changes in the gut microbiota according to dietary intake at the genus level are shown in Fig. 5 and Fig. 6. The B-diet group showed an increase in the abundance of Bacteroides of $23.4 \%(P<0.05)$ and a significant decrease in the abundance of Blautia of $45.6 \%(P<0.01)$ compared to baseline levels. In addition, the abundances of Anaerostipes $(P<0.01)$, Lachnoclostridium $(P<0.05)$, Dorea $(P<0.05)$,

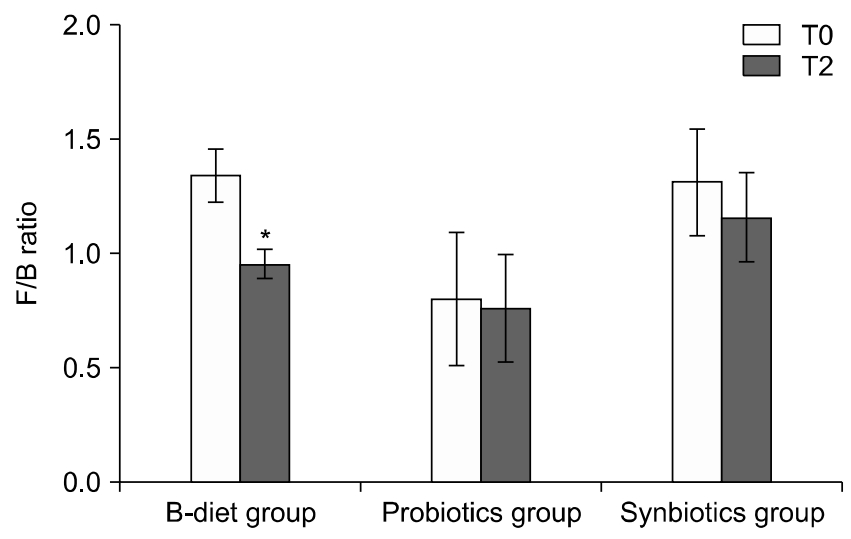

Fig. 3. Effect of each diet on changes in the Firmicutes-toBacteroidetes (F/B) ratio. T0, baseline value; T2, after 2 weeks of intervention. Values are expressed as the mean \pm SEM. B-diet group, whole grains and vegetable-enriched diet; probiotics group, probiotics capsule containing Lactobacillus plantarum PMO 08; synbiotics group, whole grains and vegetable-enriched diet combined with $L$. plantarum PMO 08. ${ }^{*} P<0.05$ compared with baseline (TO), calculated using paired $t$-tests.

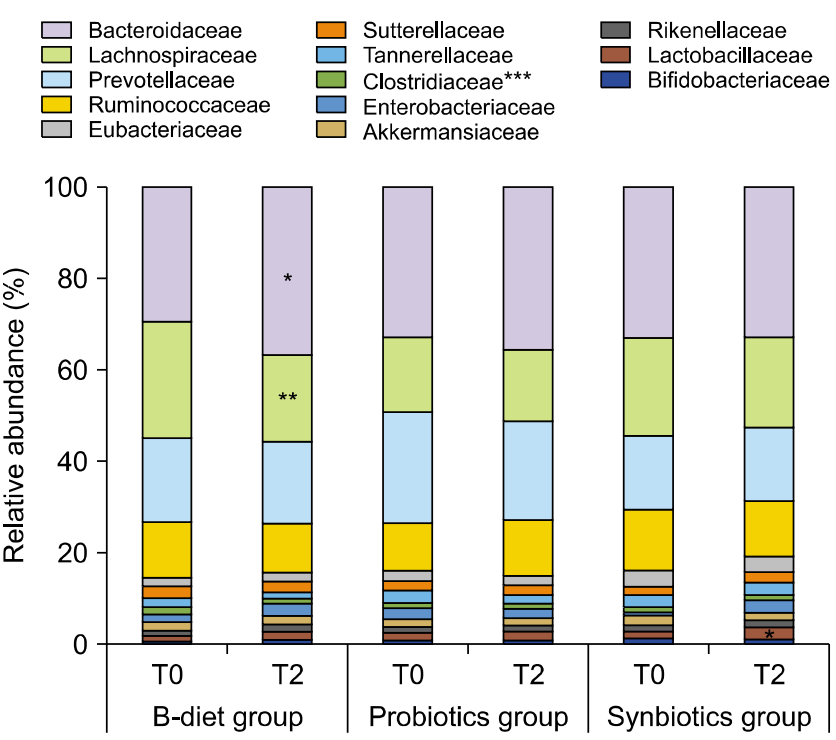

Fig. 4. Effect of each diet on relative bacterial abundances at the family level. T0, baseline value; T2, after 2 weeks of consumption. B-diet group, whole grains and vegetable-enriched diet; probiotics group, probiotics capsule containing Lactobacillus plantarum PMO 08; synbiotics group, whole grains and vegetable-enriched diet combined with $L$. plantarum $\mathrm{PMO} 08 .{ }^{*} P<$ $0.05,{ }^{* *} P<0.01$, and ${ }^{* * *} P<0.001$ compared with baseline (TO), calculated using paired $t$-tests. 
Cuneatibacter $(P<0.05)$, and Merdimonas $(P<0.05)$ were significantly decreased in the B-diet group (Fig. 6). Probiotics alone had little effect on Bacteroides and Ruminococcus levels, which are the major genera in the intestines, with the abundances of Prevotella only decreasing by

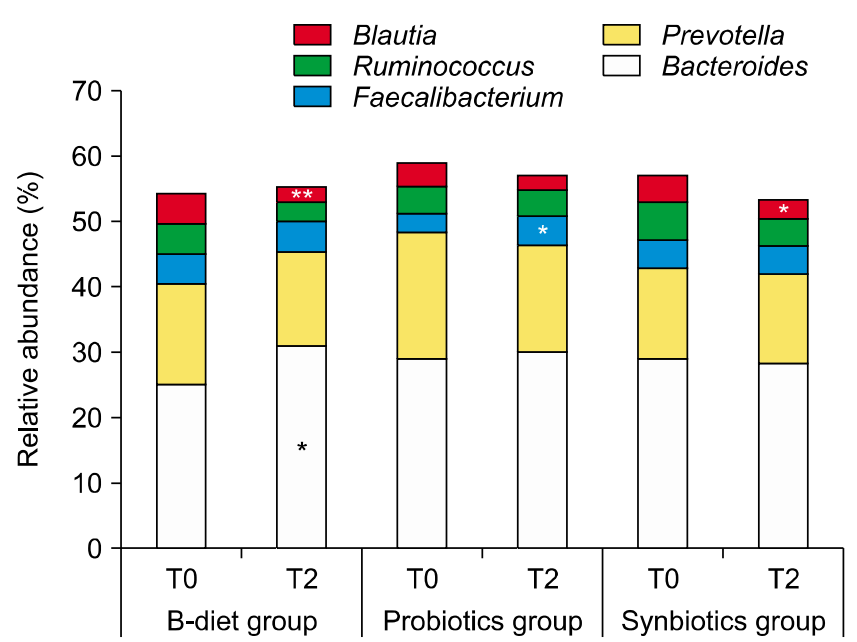

Fig. 5. Effect of each diet on relative abundances of bacteria in the major microbiota at the genus level. Relative abundances of the top five genera are shown. T0, baseline value; T2, after 2 weeks of consumption. Values are expressed as the mean \pm SEM. B-diet group, whole grains and vegetable-enriched diet; probiotics group, probiotics capsule containing Lactobacillus plantarum PMO 08; synbiotics group, whole grains and vegetable-enriched diet combined with $L$. plantarum PMO 08. ${ }^{*} P<0.05$ and ${ }^{* *} P<0.01$ compared with values at baseline (TO), calculated by paired $t$-tests.
$15.6 \%$ and $38.5 \%$, respectively, in the probiotics group. However, in the probiotics group, the abundance of Faecalibacterium, a major butyrate producer, was significantly increased by $65.4 \%(P<0.05)$. In addition, the probiotics group showed significant increases in Gemmiger (difference $=0.117, P<0.05$ ). In the synbiotics group, there were few differences in the relative abundances of Bacteroides and Prevotella, and a $30.1 \%$ decrease in the relative abundance of Ruminococcus, although this was not considered statistically significant. However, participants in the synbiotics group showed a significant decrease in the abundance of Blautia $(28.3 \%, P<0.05)$ and a significant increase in the abundance of Lactobacillus $(P<0.05)$. In addition, the abundances of Hespellia $(P<0.05)$ and Phocea $(P<0.05)$ in the synbiotics group, and Intestinomas in Bdiet group $(P<0.05)$ were significantly decreased, but this finding was omitted from the data as the relative abundances were below $0.1 \%$.

\section{Relative abundances of Lactobacillus and Bifidobacterium}

The relative abundances of the genus Lactobacillus, a beneficial intestinal bacteria, are shown in Fig. 7A. In the Bdiet group, the initial (T0) relative abundance of Lactobacillus spp. was $1.00 \pm 0.31 \%$, and increased to $1.48 \pm 0.39 \%$ after 2 weeks of dietary intake (difference $=0.481, P=$ $0.09)$. In the probiotics group, the relative abundance of Lactobacillus spp. increased from $1.48 \pm 0.52 \%$ to $1.76 \pm$ $0.46 \%$ after 2 weeks (difference $=0.282, P=0.687$ ), and in the synbiotics group, the relative abundance of Lacto-
A

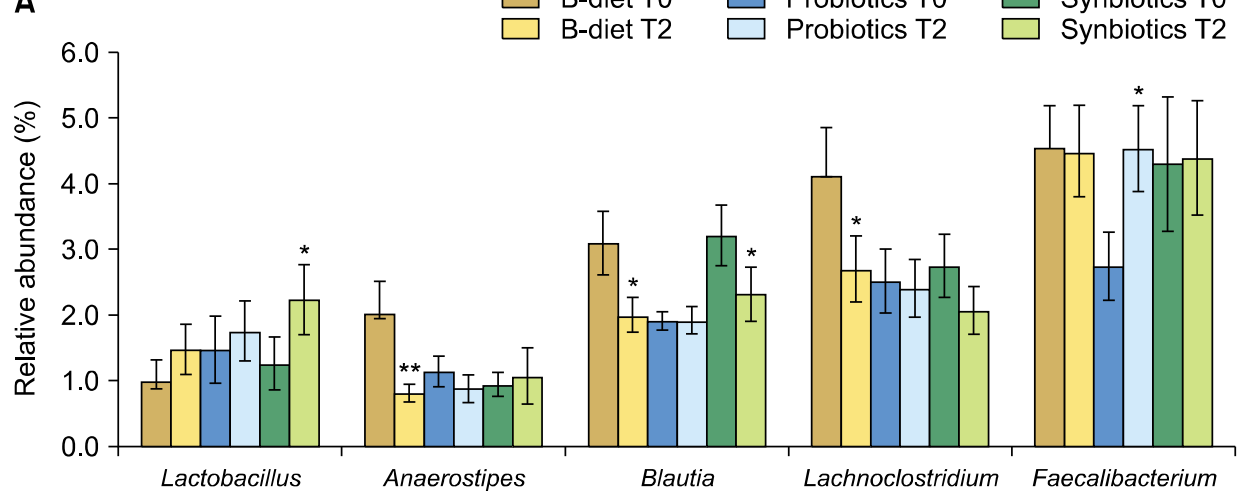

B

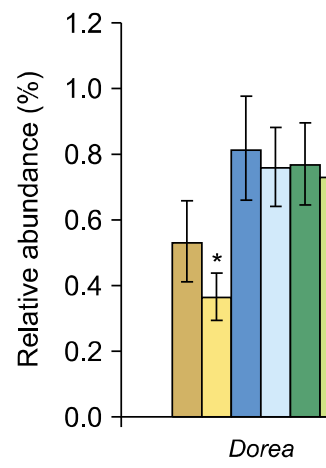

B-diet TO

TO B-diet T2

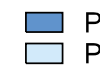

Probiotics TO Probiotics T2
Synbiotics TO Synbiotics T2

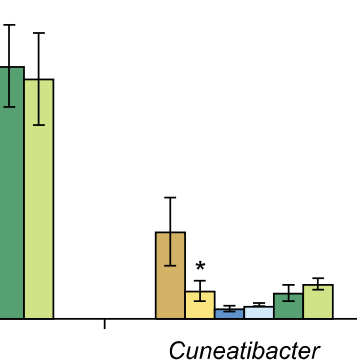

Cuneatibacter

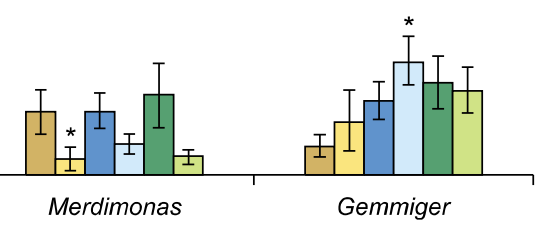

Fig. 6. Effect of each diet on relative abundances of bacteria in the $\mathrm{mi}^{-}$ crobiota at the genus level. Genera with significant differences in even one of the groups are shown. (A) Relative abundances of bacterial taxa accounting for more than $1 \%$. (B) Relative abundances of bacterial taxa accounting for less than $1 \%$. T0, baseline value; T2, after 2 weeks of consumption. Values are expressed as the mean \pm SEM. B-diet group, whole grains and vegetableenriched diet; probiotics group, probiotics capsule containing $\mathrm{Lac}^{-}$ tobacillus plantarum PMO 08; synbiotics group, whole grains and vegetable-enriched diet combined with L. plantarum PMO 08. *P<0.05 and ${ }^{* *} P<0.01$ compared with values at baseline (TO), calculated by paired $t$-tests. 
bacillus spp. increased by approximately 1.8-fold from $1.26 \pm 0.40 \%$ to $2.24 \pm 0.53 \%$ (difference $=0.981, P<0.05$ ). The relative abundances of the genus Bifidobacterium are shown in Fig. 7B. In the B-diet group, the relative abundance of Bifidobacterium spp. increased by $49.5 \%$, although this increase was not significant. No changes in the abundances of Bifidobacterium spp. were observed in either the probiotics or synbiotics groups.

\section{Changes in defecation habits induced by dietary intake}

Defecation habits of the subjects are shown in Table 4. Before the dietary intervention, the average Bristol score of the subjects was $3.9 \pm 1.20$ points, and $55.6 \%$ of the subjects scored 4 points with a normal fecal shape. An evaluation of each item (including frequency, regularity, transit time, the difficulty of defecation, incomplete evacuation, and bloating) showed that there were no significant differences between the groups at baseline (T0). The significance of dietary intake was evaluated using paired $t$-tests before and after the intervention period and showed that the "bloating" symptom was significantly alleviated in all groups. The score for bloating improved from $2.71 \pm 0.18$ to $2.10 \pm 0.18$ points $(P<0.01)$ in the B-diet group, from $2.43 \pm 0.19$ to $2.05 \pm 0.21$ points $(P<0.05)$ in
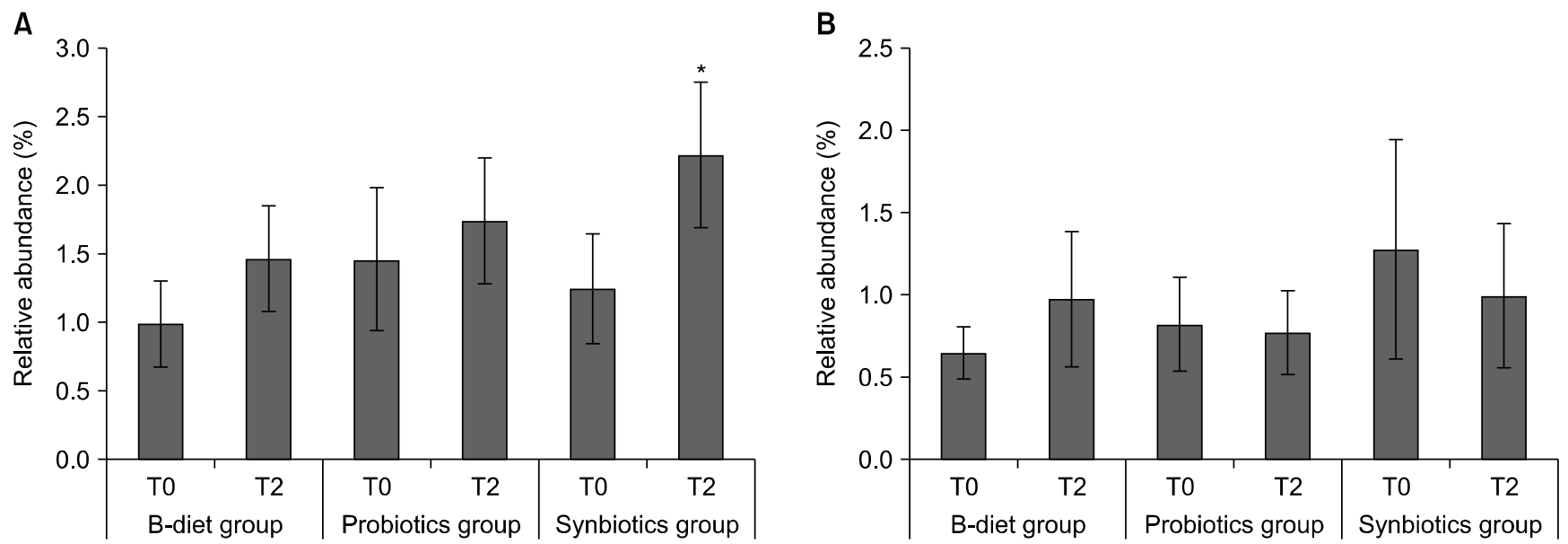

Fig. 7. Effect of each diet on (A) Lactobacillus and (B) Bifidobacterium composition of the microbiota. T0, baseline value; T2, after 2 weeks of consumption. Values are expressed as the mean \pm SEM. B-diet group, whole grains and vegetable-enriched diet; probiotics group, probiotics capsule containing Lactobacillus plantarum PMO 08; synbiotics group, whole grains and vegetable-enriched diet combined with $L$. plantarum PMO 08 . ${ }^{*} P<0.05$ compared with values at baseline (TO), calculated by paired $t$-tests.

Table 4. Defecation habits and fecal symptoms of study participants

\begin{tabular}{|c|c|c|c|c|c|c|c|}
\hline Items & Periods & B-diet group & $P$-value & Probiotics group & $P$-value & Synbiotics group & $P$-value \\
\hline \multirow[t]{2}{*}{ Bristol score } & TO & $4.24 \pm 0.23$ & 0.590 & $4.00 \pm 0.26$ & 0.760 & $3.48 \pm 0.28$ & 0.162 \\
\hline & $\mathrm{T} 2$ & $4.10 \pm 0.23$ & & $4.10 \pm 0.27$ & & $3.86 \pm 0.21$ & \\
\hline \multirow[t]{2}{*}{ Stool frequency } & TO & $3.14 \pm 0.20$ & 0.649 & $3.10 \pm 0.19$ & 1.000 & $3.19 \pm 0.19$ & 0.329 \\
\hline & $\mathrm{T} 2$ & $3.05 \pm 0.20$ & & $3.10 \pm 0.22$ & & $3.33 \pm 0.19$ & \\
\hline \multirow{2}{*}{ Stool regularity } & TO & $2.05 \pm 0.13$ & 0.666 & $2.19 \pm 0.15$ & 0.493 & $2.14 \pm 0.13$ & 0.055 \\
\hline & $\mathrm{T} 2$ & $2.00 \pm 0.14$ & & $2.10 \pm 0.15$ & & $1.86 \pm 0.14$ & \\
\hline \multirow[t]{2}{*}{ Gut transit time } & TO & $1.57 \pm 0.18$ & 0.493 & $1.48 \pm 0.11$ & 1.000 & $1.81 \pm 0.19$ & 0.379 \\
\hline & $\mathrm{T} 2$ & $1.48 \pm 0.15$ & & $1.48 \pm 0.15$ & & $1.67 \pm 0.21$ & \\
\hline \multirow[t]{2}{*}{ Difficult of defecation } & TO & $1.81 \pm 0.18$ & 0.540 & $1.86 \pm 0.16$ & 0.104 & $2.05 \pm 0.16$ & 0.030 \\
\hline & $\mathrm{T} 2$ & $1.90 \pm 0.14$ & & $1.67 \pm 0.16$ & & $1.76 \pm 0.15$ & \\
\hline \multirow[t]{2}{*}{ Incomplete evacuation } & TO & $2.29 \pm 0.18$ & 0.025 & $1.86 \pm 0.16$ & 0.056 & $2.29 \pm 0.12$ & 0.004 \\
\hline & $\mathrm{T} 2$ & $1.86 \pm 0.14$ & & $1.62 \pm 0.16$ & & $1.81 \pm 0.16$ & \\
\hline \multirow[t]{2}{*}{ Bloating } & TO & $2.71 \pm 0.18$ & 0.009 & $2.43 \pm 0.19$ & 0.042 & $2.71 \pm 0.18$ & 0.038 \\
\hline & $\mathrm{T} 2$ & $2.10 \pm 0.18$ & & $2.05 \pm 0.21$ & & $2.24 \pm 0.15$ & \\
\hline
\end{tabular}

Values are expressed as the mean \pm SD ( $n=21)$.

Significant differences were measured using paired $t$-tests.

The seven types of Bristol stool scale are as follows: type 1, separate hard lumps; type 2, lumpy and sausage-like; type 3, sausage-shape with cracks on the surface; type 4, like a smooth, soft sausage or snake; type 5, soft blobs with clear-cut edges; type 6, mushy consistency with ragged edges; type 7, liquid consistency with no solid pieces.

Constipated, including type 1 and type 2 on the Bristol stool scale; normal, including type 3, type 4, and type 5 on the Bristol stool scale; inflammation, including type 6 and type 7 on the Bristol stool scale.

B-diet group, whole grains, and vegetable-enriched diet group; probiotics group, Lactobacillus plantarum PMO 08-administered group; synbiotics group, whole grains, and vegetable-enriched diet group combined with L. plantarum PMO 08. 
the probiotics group, and from $2.71 \pm 0.18$ to $2.24 \pm 0.15$ points $(P<0.05)$ in the synbiotics group. Additionally, in the B-diet group, incomplete evacuation $(P<0.05)$ was improved, and in the synbiotics group, incomplete evacuation $(P<0.01)$ and difficulty in defecation $(P<0.05)$ were improved, indicating a positive synergistic effect. Moreover, the regularity of defecation was relatively improved $(P=0.055)$ in the synbiotics group. However, no significant changes in fecal shape (Bristol score), frequency, or transit time were observed for any of the three groups compared with baseline.

\section{Dietary compliance}

Through surveying intake of the diet provided during the experiment and any side effects, all participants were found to have complied with the provided diets and probiotic supplements during the study period, and no participants experienced any side effects (data not shown).

\section{DISCUSSION}

This study was conducted to evaluate the effect of a nutritionally balanced diet enriched in whole grains and vegetables alone or in combination with probiotics on the composition of the gut microbiota. Nutritionally balanced diets were provided to participants in lunch boxes. Meals provided on weekdays contained $561 \mathrm{kcal}$, with a ratio (\%) of carbohydrate to protein to fat of 63:16:21 and 14 $\mathrm{g}$ of dietary fiber. Meals provided on weekends contained $592 \mathrm{kcal}$, with a ratio (\%) of carbohydrates to protein to fat of 57:19:24 with $4 \mathrm{~g}$ of dietary fiber. We evaluated the synergistic effect of a nutritionally balanced diet in combination with a probiotic supplement containing $L$. plantarum PMO 08. The study showed that consumption of a nutritionally balanced diet decreased the $\mathrm{F} / \mathrm{B}$ ratio and induced changes in major genera, including an increase in the abundance of Bacteroides and decreases in the abundances of Blautia, Lachnoclostridium, and Dorea, which belong to the family Lachnospiraeceae (phylum Firmicutes, class Clostridia).

Firmicutes and Bacteroidetes account for more than $90 \%$ of the gut microbiota, and a high F/B ratio is associated with IBD, type 2 diabetes, and obesity (Magne et al., 2020). The F/B ratio is an important indicator of body weight control as it is higher in patients with obesity and decreases with reduced caloric intake (Turnbaugh et al., 2006). Ley et al. (2006) first identified a causal relationship between the microbiome and obesity, which was confirmed by inducing obesity in lean mice via transplanting the feces of obese mice, showing that the ability to extract energy from food could be affected by intestinal microorganisms. Therefore, the nutritionally balanced diet used in the current study is expected to help prevent chronic diseases by improving the composition of the gut microbiota.

In this study, the decrease in the F/B ratio induced by intake of a nutritionally balanced diet was due, in some cases, to a significant increase in the abundance of the genus Bacteroides. Bacteroides comprises Gram-negative bacteria that account for up to approximately $30 \%$ of the human fecal microbiota, and are present in feces at a concentration of $10^{9} \sim 10^{11} \mathrm{CFU} / \mathrm{g}$ (Tan et al., 2019). Bacteroides appears to have anti-obesity effects, such as inhibition of fat absorption and blood sugar, regulation of insulin and leptin levels, and improvement in immune parameters related to being overweight (Chang et al., 2019). It can possibly be used to treat and prevent osteoporosis by improving bone density through the production of vitamin K (Fujita et al., 2012). In addition, low abundances of Bacteroides coprophilus in patients with multiple sclerosis and cardiac fibrillation (Mirza et al., 2020; Xu et al., 2020) were reported in case-control studies. Indeed, the antibiotic diarrhea-prevention effects of Bacteroides fragilis ZY312 28 (Zhang et al., 2018), anticancer and immune boosting effects of Bacteroides xylanisolvens DSM 23964 (Ulsemer et al., 2016), and metabolic syndromerelated function of Bacteroides uniformis CECT7771 studied for use as probiotics (Gauffin Cano et al., 2012). In this study, the abundance of Bacteroides increased from $25.1 \%$ to $30.9 \%$ after consumption of a nutritionally balanced diet $(P<0.05)$. Bacteroides has a powerful polysaccharide breakdown system and provides nutrients and energy for the human body through breakdown of short chain fatty acids (Tan et al., 2019). The nutritionally balanced diet consisting of whole grains and vegetables is believed to have promoted the growth of Bacteroides by providing polysaccharides, such as glycan. Follow-up studies are needed to determine which dietary ingredients or nutrients in the diet contributed to the increase in the proportion of Bacteroides.

In the B-diet group, the abundance of the phylum Firmicutes, associated with obesity, type 2 diabetes, and IBD decreased, which we attributed to the significant reduction in the abundances of Clostridiaceae $(P<0.001)$ and Lachnospiraceae $(P<0.01)$, which belong to the class Clostridia and the order Clostridiales. It was reported that the abundance of Clostridiales is significantly increased in an animal model of high-fat diet-induced obesity (de La Serre et al., 2010) and can be reduced by high levels of vegetable intake (Kimura et al., 2013). Lachnospiraceae includes microorganisms associated with obesity, diabetes, and cardiovascular disease (Larsen et al., 2010; Cho and Blaser, 2012; Kameyama and Itoh, 2014; Liu et al., 2018). Kameyama and Itoh (2014) reported that the family Lachnospiraceae causes obesity and diabetes by isolating Lachnospiraceae microorganisms from hyperglycemic obese mice and injecting them into germ- 
free mice to observe increases in fasting blood sugar and adipose tissue weight, and low insulin levels. The abundance of the family Lachnospiraceae is high in patients with obesity (Cho and Blaser, 2012). High levels of Lachnospiraceae in the microbiota have been proposed to be a predictor of cardiovascular disease as the bacteria accelerate the progression of diabetes and arteriosclerosis (Larsen et al., 2010; Liu et al., 2018). Blautia, the most abundant genus in the Lachnospiraceae family, is an acetic acid-producing microorganism that is highly associated with glucose tolerance and hypertension (Kakiyama et al., 2013). When analyzing the composition of the gut microbiota according to the presence or absence of metabolic diseases in patients with obesity, Blautia spp. have been positively correlated with waist circumference, BMI, low-density lipoprotein cholesterol, and triglycerides, and have been used as diagnostic markers to predict metabolic diseases (Ohigashi et al., 2013). Dorea spp. are positively correlated with secondary bile acids, such as deoxycholic and lithocholic acids production, body weight, and total cholesterol levels in the blood (Holscher et al., 2018; Zeng et al., 2019). A high-fat/low-fiber Westerntype diet increases the levels of secondary bile acids produced by the gut microbiota, which induces intestinal cytotoxicity and ultimately leads to colon cancer or bowel diseases (O'Keefe, 2016). Thus, the reduction of Dorea spp. by intake of a nutritionally balanced diet is considered to be helpful in improving the intestinal environment.

In this study, intake of L. plantarum PMO 08 improved the gut microbiota by significantly increasing the abundance of Faecalibacterium, a beneficial bacterium found in the intestines, and decreasing the abundance of the family Clostridiaceae. Faecalibacterium is an anaerobic microorganism that produces butyrate and is present at abundances of approximately $5 \%$ in the gut microbiota of healthy adults (Martín et al., 2017). The abundance of Faecalibacterium is relatively low in weak elderly individuals and patients with obesity, irritable bowel syndrome, irritable colitis, colon cancer, or celiac disease (Martín et al., 2017). F. prausnitzii produces anti-inflammatory substances, such as shikimic and salicylic acids (Miquel et al., 2015), and induces Toll-like receptor 2-dependent immunomodulatory actions through interleukin (IL)-12 and IL-10 cytokines (Rossi et al., 2015). In an animal model of Escherichia coli-induced infection, administration of $L$. plantarum ZLP001 significantly increased the abundance of $F$. prausnitzii, increased the expression of barrierenhancing proteins [claudin-1, occludin, and zonula occludens-1 (ZO-1)], and exerted an immunomodulatory effect on the expression of pro-inflammatory cytokines, such as IL- 6 and tumor necrosis factor- $\alpha$ (Wang et al., 2018). In a mouse model of loperamide-induced constipation, administration of complex probiotics, including
L. plantarum, suppressed the decrease in stool water content due to constipation and resulted in a significant increase in the abundance of $F$. prausnitzii in the intestines (Eor et al., 2019). L. plantarum PMO 08 used in the current study may enhance barrier function, as it has been shown to increase ZO-1 expression in an obese mouse model (Oh et al., 2019) and exhibit antipruritic effects in a mouse model of drug-induced hypersensitivity (Jang et al., 2011b). Therefore, the gut barrier-enhancing function and immunomodulatory effects of $L$. plantarum PMO 08 may be explained in part by recovery of $F$. prausnitzii in the gut microbiota; however, further studies are needed to confirm this finding.

In the current study, a nutritionally balanced diet and probiotic supplements had a synergetic effect on significantly increasing the abundance of Lactobacillus spp. Moreover, in the synbiotics group, improvements were observed in defecation in addition to bloating and incomplete evacuation, which were also present in the B-diet group. Abdominal bloating might have been reduced by decreased levels of the gases $\mathrm{CO}_{2}, \mathrm{CH}_{4}$, and $\mathrm{H}_{2}$ produced by intestinal microbes, and reduced stagnation of these gases due to intestinal motility promoted by propionic acid and acetic acid produced by intestinal microbes. Improved intestinal motility could have also improved the difficulty of defecation (Eor et al., 2019).

In this study, we conducted an analysis of gut microbiota according to dietary intake in healthy adults. Although the gut microbiota was not in a state of dysbiosis, replacing one meal per day for 2 weeks induced positive changes in the composition of the gut microbiota. However, since we did not measure hematological indicators, only limited physiological changes induced by alterations to the composition of the gut microbiota were observed. Therefore, to confirm the effect of a nutritionally balanced diet and probiotic supplementation on the human body, follow-up studies are needed to analyze the metabolites and physiological indicators together.

The objective of this study was to evaluate the effects of a nutritionally balanced diet consisting of whole grains and vegetables alone or in combination with probiotic supplements on the gut microbiota of healthy adults. Replacing one meal per day with a nutritionally balanced diet improved the intestinal environment by significantly lowering the $\mathrm{F} / \mathrm{B}$ ratio, decreasing major gut microbiota, such as those of the families Clostridiaceae and Lachnospiraceae and the genera Blautia, Dorea, and Lachnoclostridium, and alleviating symptoms, such as bloating and incomplete evacuation. L. plantarum PMO 08 intake improved the gut microbiota by increasing the abundance of Faecalibacterium, Ruminococcus, and Gemmiger, and decreasing the abundance of the family Clostridiaceae. The synergetic effects of a nutritionally balanced diet in combination with probiotic supplementation were confirmed 
by an increase in the abundance of Lactobacillus and improvements in bloating, incomplete evacuation, and difficulty of defecation. To our knowledge, this study is the first to show that a nutritionally balanced diet consisting of whole grains and vegetables may positively change the composition of the gut microbiota. Importantly, our results may support the design of healthy balanced diets to control the gut microbiota. In addition, this study shows that $L$. plantarum PMO 08 can be safely used as a probiotic supplement as it promotes colonization of beneficial bacteria and inhibits harmful bacteria without any side effects in humans.

\section{AUTHOR DISCLOSURE STATEMENT}

Y.J.O., K.N., Y.K., S.Y.L., J.I.K, and S.Y.L. are employees of Pulmuone Co., Ltd.. H.S.K. and K.T.H. declare no conflict of interest.

\section{REFERENCES}

Arumugam M, Raes J, Pelletier E, Le Paslier D, Yamada T, Mende DR, et al. Enterotypes of the human gut microbiome. Nature. 2011. 473:174-180.

Barrett HL, Gomez-Arango LF, Wilkinson SA, McIntyre HD, Callaway LK, Morrison M, et al. A vegetarian diet is a major determinant of gut microbiota composition in early pregnancy. Nutrients. 2018. 10:890. https://doi.org/10.390/10070890

Cândido FG, Valente FX, Grześkowiak ŁM, Moreira APB, Rocha DMUP, Alfenas RCG. Impact of dietary fat on gut microbiota and low-grade systemic inflammation: mechanisms and clinical implications on obesity. Int J Food Sci Nutr. 2018. 69:125143.

Chae L. The effects of Korean-DASH diet on metabolic syndrome, insulin resistance and gut microbiota. Master's thesis. Seoul National University, Seoul, Korea. 2016.

Chang CJ, Lin TL, Tsai YL, Wu TR, Lai WF, Lu CC, et al. Next generation probiotics in disease amelioration. J Food Drug Anal. 2019. 27:615-622.

Cho I, Blaser MJ. The human microbiome: at the interface of health and disease. Nat Rev Genet. 2012. 13:260-270.

De Angelis M, Garruti G, Minervini F, Bonfrate L, Portincasa P, Gobbetti M. The food-gut human axis: the effects of diet on gut microbiota and metabolome. Curr Med Chem. 2019. 26:35673583.

de La Serre CB, Ellis CL, Lee J, Hartman AL, Rutledge JC, Raybould HE. Propensity to high-fat diet-induced obesity in rats is associated with changes in the gut microbiota and gut inflammation. Am J Physiol Gastrointest Liver Physiol. 2010. 299:G440G448.

Eor JY, Tan PL, Lim SM, Choi DH, Yoon SM, Yang SY, et al. Laxative effect of probiotic chocolate on loperamide-induced constipation in rats. Food Res Int. 2019. 116:1173-1182.

FAO/WHO. Report of a joint FAO/WHO expert consultation on evaluation of health and nutritional properties of probiotics in food including powder milk with live lactic acid bacteria. 2001 [cited 2020 Nov 10]. Available from: http://www.fao.org/ /a0512e/a0512e.pdf

Fujita Y, Iki M, Tamaki J, Kouda K, Yura A, Kadowaki E, et al. Association between vitamin $\mathrm{K}$ intake from fermented soybeans, natto, and bone mineral density in elderly Japanese men: the Fujiwara-kyo Osteoporosis Risk in Men (FORMEN) study. Osteoporos Int. 2012. 23:705-714.

Garcia-Mantrana I, Selma-Royo M, Alcantara C, Collado MC. Shifts on gut microbiota associated to mediterranean diet adherence and specific dietary intakes on general adult population. Front Microbiol. 2018. 9:890. https://doi.org/10.3389/ fmicb.2018.00890

Gauffin Cano P, Santacruz A, Moya Á, Sanz Y. Bacteroides uniformis CECT 7771 ameliorates metabolic and immunological dysfunction in mice with high-fat-diet induced obesity. PLoS One. 2012. 7:e41079. https://doi.org/10.1371/journal.pone.041079

Ha K, Nam K, Song Y. A moderate-carbohydrate diet with plant protein is inversely associated with cardiovascular risk factors: the Korea National Health and Nutrition Examination Survey 2013-2017. Nutr J. 2020. 19:84. https://doi.org/10.1186/ 12937-20-00603-2

Hills RD Jr, Pontefract BA, Mishcon HR, Black CA, Sutton SC, Theberge CR. Gut microbiome: profound implications for diet and disease. Nutrients. 2019. 11:1613.

Holscher HD, Guetterman HM, Swanson KS, An R, Matthan NR, Lichtenstein $\mathrm{AH}$, et al. Walnut consumption alters the gastrointestinal microbiota, microbially derived secondary bile acids, and health markers in healthy adults: a randomized controlled trial. J Nutr. 2018. 148:861-867.

Jang SE, Hyun YJ, Oh YJ, Choi KB, Kim T, Yeo IH, et al. Adhesion activity of Lactobacillus plantarum PM 008 isolated from kimchi on the intestine of mice. J Bacteriol Virol. 2011a. 41:83-90.

Jang SE, Hyun YJ, Trinh HT, Han MJ, Kim DH. Anti-scratching behavioral effect of Lactobacillus plantarum PM008 isolated from kimchi in mice. Immunopharmacol Immunotoxicol. 2011b. 33:539-544.

Kakiyama G, Pandak WM, Gillevet PM, Hylemon PB, Heuman DM, Daita K, et al. Modulation of the fecal bile acid profile by gut microbiota in cirrhosis. J Hepatol. 2013. 58:949-955.

Kameyama K, Itoh K. Intestinal colonization by a Lachnospiraceae bacterium contributes to the development of diabetes in obese mice. Microbes Environ. 2014. 29:427-430.

Kimura I, Ozawa K, Inoue D, Imamura T, Kimura K, Maeda T, et al. The gut microbiota suppresses insulin-mediated fat accumulation via the short-chain fatty acid receptor GPR43. Nat Commun. 2013. 4:1829. https://doi.org/10.1038/comms2852

Larsen N, Vogensen FK, van den Berg FW, Nielsen DS, Andreasen AS, Pedersen BK, et al. Gut microbiota in human adults with type 2 diabetes differs from non-diabetic adults. PLoS One. 2010. 5:e9085. https://doi.org/10.1371/journal.one.0009085

Lee ES, Song EJ, Nam YD, Lee SY. Probiotics in human health and disease: from nutribiotics to pharmabiotics. J Microbiol. 2018. 56:773-782

Lewis SJ, Heaton KW. Stool form scale as a useful guide to intestinal transit time. Scand J Gastroenterol. 1997. 32:920-924.

Ley RE, Turnbaugh PJ, Klein S, Gordon JI. Microbial ecology: human gut microbes associated with obesity. Nature. 2006. 444: 1022-1023.

Liu B, Zhang Y, Wang R, An Y, Gao W, Bai L, et al. Western diet feeding influences gut microbiota profiles in apoE knockout mice. Lipids Health Dis. 2018. 17:159. https://doi.org/10.186/ 12944-018-0811-8

Longstreth GF, Thompson WG, Chey WD, Houghton LA, Mearin F, Spiller RC. Functional bowel disorders. Gastroenterology. 2006. 130:1480-1491.

Losasso C, Eckert EM, Mastrorilli E, Villiger J, Mancin M, Patuzzi $\mathrm{I}$, et al. Assessing the influence of vegan, vegetarian and omnivore oriented westernized dietary styles on human gut microbiota: a cross sectional study. Front Microbiol. 2018. 9:317. https://doi.org/10.3389/fmicb.2018.00317

Magne F, Gotteland M, Gauthier L, Zazueta A, Pesoa S, Navarrete 
$\mathrm{P}$, et al. The firmicutes/bacteroidetes ratio: a relevant marker of gut dysbiosis in obese patients?. Nutrients. 2020. 12:1474. https://doi.org/10.3390/nu12051474

Martín R, Miquel S, Benevides L, Bridonneau C, Robert V, Hudault $S$, et al. Functional characterization of novel Faecalibacterium prausnitzii strains isolated from healthy volunteers: a step forward in the use of $F$. prausnitzii as a next-generation probiotic. Front Microbiol. 2017. 8:1226. https://doi.org/10.3389/ fmicb.2017.01226

Miquel S, Leclerc M, Martin R, Chain F, Lenoir M, Raguideau S, et al. Identification of metabolic signatures linked to anti-inflammatory effects of Faecalibacterium prausnitzii. mBio. 2015. 6:e00300-15. https://doi.org/10.1128/mBio.00300-15

Mirza A, Forbes JD, Zhu F, Bernstein CN, Van Domselaar G, Graham M, et al. The multiple sclerosis gut microbiota: A systematic review. Mult Scler Relat Disord. 2020. 37:101427. https://doi.org/10.1016/j.msard.2019.101427

O'Keefe SJ. Diet, microorganisms and their metabolites, and colon cancer. Nat Rev Gastroenterol Hepatol. 2016. 13:691-706.

Oh YJ, Kim HJ, Kim TS, Yeo IH, Ji GE. Effects of Lactobacillus plantarum PMO 08 alone and combined with chia seeds on metabolic syndrome and parameters related to gut health in high-fat diet-induced obese mice. J Med Food. 2019. 22:1199-1207.

Oh YJ, Kim TS, Moon HW, Lee SY, Lee SY, Ji GE, et al. Lactobacillus plantarum $\mathrm{PMO} 08$ as a probiotic starter culture for plant-based fermented beverages. Molecules. 2020. 25:5056. https://doi. org/0.3390/molecules 25215056

Ohigashi S, Sudo K, Kobayashi D, Takahashi O, Takahashi T, Asahara $\mathrm{T}$, et al. Changes of the intestinal microbiota, short chain fatty acids, and fecal $\mathrm{pH}$ in patients with colorectal cancer. Dig Dis Sci. 2013. 58:1717-1726.

Rossi O, Khan MT, Schwarzer M, Hudcovic T, Srutkova D, Duncan $\mathrm{SH}$, et al. Faecalibacterium prausnitzii strain HTF-F and its extracellular polymeric matrix attenuate clinical parameters in DSSinduced colitis. PLoS One. 2015. 10:e0123013. https://doi. org/10.1371/journal.pone.0123013

Schoeler M, Caesar R. Dietary lipids, gut microbiota and lipid metabolism. Rev Endocr Metab Disord. 2019. 20:461-472.

Shin JH, Jung S, Kim SA, Kang MS, Kim MS, Joung H, et al. Differential effects of typical korean versus american-style diets on gut microbial composition and metabolic profile in healthy overweight Koreans: a randomized crossover trial. Nutrients.
2019. 11:2450. https://doi.org/10.3390/u11102450

Shokryazdan P, Faseleh Jahromi M, Liang JB, Ho YW. Probiotics: from isolation to application. J Am Coll Nutr. 2017. 36:666676.

Singh RK, Chang HW, Yan D, Lee KM, Ucmak D, Wong K, et al. Influence of diet on the gut microbiome and implications for human health. J Transl Med. 2017. 15:73. https://doi.org/10. 1186/s12967-017-1175-y

Tan H, Zhai Q, Chen W. Investigations of Bacteroides spp. towards next-generation probiotics. Food Res Int. 2019. 116:637-644.

Turnbaugh PJ, Ley RE, Mahowald MA, Magrini V, Mardis ER, Gordon JI. An obesity-associated gut microbiome with increased capacity for energy harvest. Nature. 2006. 444:10271031.

Ulsemer P, Toutounian K, Kressel G, Goletz C, Schmidt J, Karsten $\mathrm{U}$, et al. Impact of oral consumption of heat-treated Bacteroides xylanisolvens DSM 23964 on the level of natural TF $\alpha$-specific antibodies in human adults. Benef Microbes. 2016. 7:485-500.

Vangay P, Johnson AJ, Ward TL, Al-Ghalith GA, Shields-Cutler RR, Hillmann BM, et al. US Immigration westernizes the human gut microbiome. Cell. 2018. 175:962-972.e10.

Wang J, Ji H, Wang S, Liu H, Zhang W, Zhang D, et al. Probiotic Lactobacillus plantarum promotes intestinal barrier function by strengthening the epithelium and modulating gut microbiota. Front Microbiol. 2018. 9:1953. https://doi.org/10.3389/micb. 018.01953

Xu F, Fu Y, Sun TY, Jiang Z, Miao Z, Shuai M, et al. The interplay between host genetics and the gut microbiome reveals common and distinct microbiome features for complex human diseases. Microbiome. 2020. 8:145. https://doi.org/10.1186/ 40168-020-00923-9

Yeo IK, Kim TS, Tae SG. Microorganism, Lactobacillus plantarum PMO08 (KFCC-11028), decreasing blood cholesterol concentration. KR Patent 10-0264361. 2000.

Zeng Q, Li D, He Y, Li Y, Yang Z, Zhao X, et al. Discrepant gut microbiota markers for the classification of obesity-related metabolic abnormalities. Sci Rep. 2019. 9:13424. https://doi. org/10.1038/s41598-019-49462-w

Zhang W, Zhu B, Xu J, Liu Y, Qiu E, Li Z, et al. Bacteroides fragilis protects against antibiotic-associated diarrhea in rats by modulating intestinal defenses. Front Immunol. 2018. 9:1040. https://doi.org/10.3389/fimmu.2018.01040 